\title{
Bringing development into the equation
}

Hauser $\mathrm{TU}^{1,2}$

${ }^{1}$ Max Planck UCL Centre for Computational Psychiatry and Ageing Research, London WC1B 5EH, United Kingdom.

${ }^{2}$ Wellcome Centre for Human Neuroimaging, University College London, London WC1N 3BG, United Kingdom.

\section{Corresponding author}

Tobias U. Hauser

Max Planck UCL Centre for Computational Psychiatry and Ageing Research

University College London

10-12 Russell Square

London WC1B 5EH

United Kingdom

Phone: +44 / 2076795264

Email: t.hauser@ucl.ac.uk

Conflict of interest: The author declares no competing financial interests.

Acknowledgements: TUH is supported by a Wellcome Sir Henry Dale Fellowship (211155/Z/18/Z), a grant from the Jacobs Foundation (2017-1261-04), the Medical Research Foundation, and a 2018 NARSAD Young Investigator grant (27023) from the Brain \& Behavior Research Foundation. The Max Planck UCL Centre is a joint initiative supported by UCL and the Max Planck Society. The Wellcome Centre for Human Neuroimaging is supported by core funding from the Wellcome Trust (203147/Z/16/Z). 
Fifteen-year-old Daniel ${ }^{1}$ was recently diagnosed with obsessive-compulsive disorder (OCD). He is constantly afraid that might have lost one of his belongings, maybe the phone, or the notebook, or that blue pen. Consequently, he needs to check every place he is about to leave to make sure that he has not left anything behind, under the chair, between the sofa cushions, or in the manhole in the pavement that he just walked across. Daniel's OCD is highly debilitating, whenever he needs to change places, he spends ages checking and checking again that he truly has got everything with him. This makes attending school very difficult, and the way to school has become an ordeal. As a consequence, his academic achievements have suffered, he has difficulty spending time with friends, and his OCD gives rise to substantial tension within his family. But Daniel is not alone. Juvenile OCD in fact is a relatively common but neglected psychiatric disorder. Up to $3 \%$ of the population suffer from OCD, and the majority of patients have an early onset in late childhood or early adolescence (1).

Not only OCD, but most psychiatric disorders show a sharp increase in adolescence, from mood to psychotic disorders (2). But nobody knows why adolescence is this period of heightened vulnerability for psychiatric disorders. The epidemiological data (2) clearly demonstrate that adolescence is a critical period for the emergence of psychiatric disorders, but why this is remains largely unknown. The lack of knowledge about the mechanisms underlying adolescent psychiatric disorders not only comes from the fact that doing research with juvenile patients is considerably more challenging than with adult patients, but also because adolescent mental health has long been neglected by founding bodies (3).

By ignoring adolescence as a particularly important period for mental health, we not only fail to understand and help youths that suffer from mental health problems, but we are

\footnotetext{
${ }^{1}$ Name changed
} 
also ignoring critical information that is likely to be crucial for understanding the neurocognitive mechanisms that underlie psychiatric disorders in general.

Adolescence is a period of fundamental change and adjustments. Peers and peer relationships become more and more important whilst parents and family start being frowned upon in a struggle to claim independence. Moreover, puberty forces an active examination of one's own sexual identity and the accompanying norms and expectations. Critically, all these changes are embedded and reflected in an ongoing maturation and reorganisation of cognitive and brain functions.

It is thus likely that psychiatric disorders arise due to a derailed neurocognitive maturation. This means that if neural and cognitive development starts deviating from its normative maturational trajectory, then this may cause a widespread disruption of neurocognitive maturation, which in turn can express in the emergence of psychiatric symptoms. It is thus of utmost importance to understand the normative developmental trajectories and to identify when and how they can go awry, and how they lead to mental health problems.

Over the last two decades we have studied how the brain matures and realised that brain development is not restricted to early childhood years, but lasts well into adulthood $(4,5)$. We have traced how the brain's macrostructure changes during development. We found that white matter across many brain areas steadily increases throughout youths. We also found that gray matter follows a more complex pattern with increase in size until early adolescence, when gray matter volumes start to decrease again. Moreover we have found that these processes come to a halt first in sensorimotor areas, whilst the higher cognitive areas, such as prefrontal cortex, show a substantially prolonged maturation (5). However, what we are still unclear about - and what is critical if we want to understand how development affects the computations in and across neural populations - is what the underlying, microstructural mechanisms are. Are the 
observed macrostructural effects driven by synaptic pruning (i.e. the loss of connections) or by an increase in myelination (i.e. a better insulation of connections) (5)? Whilst both processes receive some support from ex-vivo studies, understanding the main drivers is critical as they have vastly different implications for network functioning. A recent longitudinal study has shed some light onto the ongoing microstructural processes during adolescence (6). Using advanced neuroimaging methods that closely reflect brain myelin, we found that myelin content steadily increases throughout adolescence across both gray and white matter. Importantly, there was substantial heterogeneity of myelin growth between adolescents, and those differences were related to their mental health symptoms. Adolescents that scored high on OCD traits showed a reduced ongoing myelin-related growth in specific fronto-striatal loops, whilst impulsive adolescents had a reduced growth in inferior frontal and surrounding areas (6). The latter area was also predictive for an ongoing change in their symptoms, where the adolescents with the least myelin-related growth were the ones that became more impulsive throughout the period of the study (6). These findings highlight the importance for carefully tracing brain development, which will allow us to gain insights about the emergence of psychiatric symptoms.

A further challenge that lies ahead of us is to meaningfully link neuroanatomical changes and psychiatric symptoms. How is it that a brain area with a specific neuroanatomical alteration can cause the profound and complex symptoms of a psychiatric disorder? Neuroanatomy studies often either take an agnostic approach by simply stating a link with no interpretation about how an alteration in one specific brain area leads to the expression of symptoms, or they retreat to reverse inference highlighting the role of a given brain area in some symptom-related function. However, bridging the gap between neuroanatomy and psychiatric symptoms is non-trivial and needs to be directly addressed using targeted approaches. One promising way for bridging this gap is to understand for what cognitive 
processes a given brain area is used to, and determine how this cognitive process is affected in a given disorder. Such an approach requires that we carefully dissect the symptoms that are present in psychiatric patients and understand the computational mechanisms that give rise to these symptoms. Such computational psychiatry approaches have shown great promise across a variety of cognitive domains and disorders. In the case of OCD, multiple mechanisms have been identified that may underlie the symptoms we observe in patients. For example, the excessive habitual compulsions that are characteristic for OCD patients has been linked to a deficit in model-based reasoning, a computational process that allows the utilisation of complex representations of a task (7). Likewise, OCD patients are known to suffer from a pervasive indecisiveness and doubt. Recent computational work not only been able to capture this experimentally in the context of information gathering, but was also able to determine that this was driven by a delayed emergence of subjective costs during a deliberation process $(8,9)$. These cognitive mechanisms thus may form a useful basis to tie psychiatric symptoms to underlying neurocognitive mechanisms.

However, understanding how symptoms and neurocognitive mechanisms are linked is only a first step. Equally critical is to understand how these processes derail and lead to disorder during development. When is it that a certain neurocognitive impairment emerges during childhood and adolescence? And does this predict the later emergence of psychiatric symptoms. If we are able to observe how neurocognitive mechanisms and psychiatric symptoms develop and derail, then we will be able to establish a functional directionality and come closer to a true causal understanding of how brain function and mental health problems are linked. This, however, necessitates considerable efforts and meticulous longitudinal studies, in which we trace the ongoing development of both neurocognitive mechanisms and psychiatric symptoms. This developmental computational psychiatry approach (10) is still in 
its infancy, from many neurocognitive mechanisms we do not even yet know their normative development in healthy youths.

By bringing development into the equation, we will not only be able to understand how neurocognitive processes and psychiatric symptoms impact on each other, but we may also be able to detect evolving impairments early before actual psychiatric disorders manifest. A Developmental Computational Psychiatry thus could help us develop early interventions and reduce the emergence of psychiatric disorders during adolescence. 


\section{References}

1. Shafran R (2003): Obsessive-compulsive disorder in children and adolescents. ObsessiveCompulsive Disorder: Theory, Research and Treatment. Wiley.

2. Kessler RC, Berglund P, Demler O, Jin R, Merikangas KR, Walters EE (2005): Lifetime prevalence and age-of-onset distributions of DSM-IV disorders in the National Comorbidity Survey Replication. Arch Gen Psychiatry 62: 593-602.

3. Woelbert E, Kirtley A, Balmer N, Dix S (2019): How much is spent on mental health research: developing a system for categorising grant funding in the UK. Lancet Psychiatry 6: 445-452.

4. Paus T, Keshavan M, Giedd JN (2008): Why do many psychiatric disorders emerge during adolescence? Nat Rev Neurosci 9: 947-957.

5. Paus T (2010): Growth of white matter in the adolescent brain: myelin or axon? Brain Cogn 72: $26-35$.

6. Ziegler G, Hauser TU, Moutoussis M, Bullmore ET, Goodyer IM, Fonagy P, et al. (2019): Compulsivity and impulsivity traits linked to attenuated developmental frontostriatal myelination trajectories. Nat Neurosci 22: 992-999.

7. Gillan CM, Kalanthroff E, Evans M, Weingarden HM, Jacoby RJ, Gershkovich M, et al. (2020): Comparison of the Association Between Goal-Directed Planning and Selfreported Compulsivity vs Obsessive-Compulsive Disorder Diagnosis. JAMA Psychiatry 77: 77-85.

8. Hauser TU, Moutoussis M, Iannaccone R, Brem S, Walitza S, Drechsler R, et al. (2017): Increased decision thresholds enhance information gathering performance in juvenile Obsessive-Compulsive Disorder (OCD). PLOS Comput Biol 13: e1005440. 
9. Hauser TU, Moutoussis M, Dayan P, Dolan RJ (2017): Increased decision thresholds trigger extended information gathering across the compulsivity spectrum. Transl Psychiatry 7: 1296.

10. Hauser TU, Will G-J, Dubois M, Dolan RJ (2019): Annual Research Review: Developmental computational psychiatry. $J$ Child Psychol Psychiatry. https://doi.org/10.1111/jcpp.12964 